\title{
Resource Allocation Mechanisms in Satellite Cooperative Systems
}

\author{
Luca Simone Ronga and Rosalba Suffritti \\ CNIT \\ Via di S. Marta 3, Firenze 50139, Italy \\ phone: +39055 4796485, fax: +39055 472858 \\ E-mail: \{luca.ronga, rosalba.suffritti\}@cnit.it
}

\author{
Enrico Del Re \\ University of Florence \\ Electronics and Telecommunications Department \\ Via di S. Marta 3, Firenze 50139, Italy \\ phone: +39055 4796285, fax: +39055 472858 \\ E-mail: $\{$ enrico.delre\}@unifi.it
}

\begin{abstract}
This paper reports a study concerning the assessment of cooperation effects at MAC layer in DVB-RCS systems. In particular, a modified resource allocation mechanism has been considered in order to implement a Selective Forwarding cooperation scheme within a group of sources working in a landvehicular scenario. The achieved results are presented in terms of aggregated average throughput for different source loads. The use of cooperation can allow improving system performance depending on the number of cooperators considered and the different channel conditions which they are subject to, and increasing the number of scenarios characterized by different propagation conditions wherein the system can transmit data packets compared to the case of absence of cooperation.
\end{abstract}

\section{INTRODUCTION}

Cooperative strategies can be very helpful if adopted in scenarios characterised by continuous occurrence of LOS (Lineof-sight) and NLOS (Non-line-of-sight) channel conditions and, therefore, it can be interesting to assess their implementation in critical satellite contexts, such as, for example, the mobile satellite one.

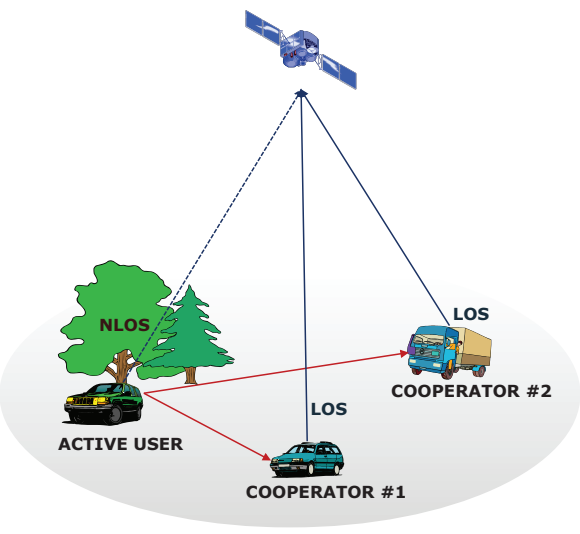

Fig. 1. Cooperative satellite scenario

As shown in Fig. 1, a user, called "active" because it needs to transmit its own informative data through the satellite, encounters bad channel conditions but it can be helped in its transmission by other users belonging to its cluster, called "cooperators" which, instead, see the satellite channel in better conditions.

However, the adoption of cooperation is a critical resource allocation issue. In fact, considering a star topology system based on DVB-RCS (Digital Video Broadcasting - Return Channel Satellite) standard, where a MF-TDMA (Multi Frequency - Time Division Multiple Access) scheme is employed, a certain number of frequency/timeslots are assigned by the Network Control Centre to each user within each superframe depending on the specific demand, through the transmission of the Terminal Burst Time Plan (TBTP), which is part of the forward link signalling, [1]. In case of cooperation, a portion of slots have to be reserved for cooperators so that these can retransmit, depending on the adopted cooperation scheme, informative packets of those users which, for each superframe, play the role of active users.

Therefore, it is crucial to select the proper allocation mechanism which:

- chooses the part of superframe devoted to cooperation;

- assigns cooperation slots to cooperators;

- associates the cooperators with the active users which, in that particular superframe, need to cooperate.

This paper deals with the assessment of cooperation effects at MAC layer in DVB-RCS systems. In particular, a modified resource allocation mechanism which implements a Selective Forwarding cooperation scheme, [2]-[3], within a group of sources working in a land-vehicular scenario, is considered. This cooperation scheme derives from the Decode and Forward technique and it is based on the concept that cooperators repeat active users packets by transmitting them through different channel paths with the condition that only the successfully decoded packets received from active users, are sent toward the final destination. Therefore, this strategy requires FEC (Forward Error Correction) decoding followed by a Cyclic Redundancy Check (CRC) to detect possible errors in the packets sent from the active users to the cooperators. The paper is organised as follows. Section II presents the system model focusing on the adopted traffic model and providing some details about the satellite channel model. In Section III the proposed resource allocation mechanism which encompasses the cooperation is described while in Section IV the achieved results showing the system performance in terms of aggregate average throughput are reported. Finally, some conclusions are given in Section V. 


\section{SySTEM MODEL}

The proposed model considers a group of sources (also called simply "users" in the following), uniformly distributed in a coverage area, which generate Internet traffic according to an $O N-O F F$ Model, as shown in Fig. 2, [4]-[5]. In $O N$ state, sources emit packets, each one at peak rate $\rho$, while in $O F F$ state, they stop any transmission, [6].

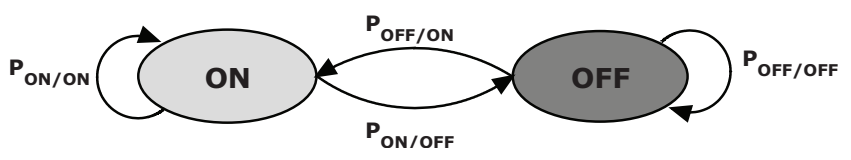

Fig. 2. ON-OFF Source Model

The bursty traffic model corresponds to an $M / G / 1$ queue, where the inter-arrival time, $t_{o f f}$, follows the exponential distribution whose the Probability Density Function (PDF) is:

$$
P D F\left(t_{o f f}\right)=\frac{1}{\mu} \exp \left(-\frac{t_{o f f}}{\mu}\right) \quad t_{o f f} \geq 0
$$

where $\mu$ is the mean of the distribution $\left(\mu=\bar{t}_{\text {off }}\right)$, while the service time, $t_{o n}$, is Pareto distributed:

$$
\operatorname{PDF}\left(t_{\text {on }}\right)= \begin{cases}\alpha \frac{\beta^{\alpha}}{t_{\text {on }}^{\alpha+1}} & t_{\text {on }} \geq \beta \\ 0 & t_{\text {on }}<\beta\end{cases}
$$

where $\alpha$, which is called shape, is an adimensional positive parameter and $\beta$, which is called scale, is the (positive) minimum possible value of $t_{o n}$ and it is, therefore, measured in seconds.

As $\alpha$ tends to infinity, the Pareto distribution approaches $\delta(x-\beta)$ where $\delta$ is the Dirac delta function.

In this model, sources can be in $O N$ state with probability $P_{o n}$ given by the following expression, [6]:

$$
P_{o n}=\frac{\bar{t}_{o n}}{\bar{t}_{o n}+\bar{t}_{\text {off }}}
$$

where $\bar{t}_{\text {on }}$ and $\bar{t}_{\text {off }}$ are the average $O N$ and $O F F$ time, respectively; and in $O F F$ state with probability $P_{\text {off }}$ given by:

$$
P_{o f f}=1-P_{o n}=\frac{\bar{t}_{o f f}}{\bar{t}_{o n}+\bar{t}_{o f f}}
$$

For the Pareto distribution, the mean value is expressed in terms of its shape parameter, $\alpha$, and its scale parameter, $\beta$, as:

$$
\bar{t}_{\text {on }}=\frac{\alpha}{\alpha-1} \beta \quad \alpha>1
$$

Therefore, considering the equations (3) and (5), the scale parameter can be calculated in function of $P_{o n}, \bar{t}_{\text {off }}$ and $\alpha$ as follows:

$$
\begin{aligned}
\beta & =\frac{\alpha-1}{\alpha} \cdot \bar{t}_{o n} \\
& =\frac{\alpha-1}{\alpha} \cdot \frac{\bar{t}_{o f f} P_{o n}}{1-P_{o n}} \\
& =f\left(P_{o n}, \alpha, \bar{t}_{o f f}\right)
\end{aligned}
$$

In this way, fixing the values of $\bar{t}_{\text {off }}$ and $\alpha, \beta$ turns to be function only of $P_{o n}$ which can be varied depending on the source load. The set of parameters considered in the simulations for each distribution is reported in Table I. The source peak rate value $\rho$ is one of peak information bit rates considered in the MF-TDMA scheme for ATM traffic timeslots in the DVB-RCS standard, [7].

\begin{tabular}{|c|c|c|c|}
\hline OFF & \multicolumn{3}{|c|}{ ON } \\
\hline$\mu=\bar{t}_{\text {off }}$ & $\alpha$ & $\beta(\mathrm{s})$ & $\rho$ \\
\hline $3.5 \mathrm{~s}$ & 1.2 & $f\left(P_{\text {on }}\right)$ & $384 \mathrm{kbit} / \mathrm{s}$ \\
\hline
\end{tabular}

TABLE I

ON/OFF MODEL PARAMETERS

In the proposed model, it is also assumed that the considered group of sources is working in a land vehicular scenario, [8]. This means that all DVR-RCS users, interconnected through terrestrial wireless links (considered ideal in this work), face a Land Mobile Satellite (LMS) channel whose characteristics are described in detail in [9]. For sake of clarity the model is summarised in the following. This considers a frequency nonselective LMS channel at $K u$ band for which a three states (LOS, Shadowed and Blocked) Markov-chain based model is proposed for the fading process. According to this class of models, the amplitude of the channel coefficient, which represents the amplitude of the fading term, is divided into fast and slow fading. Slow fading events are normally modelled as a finite state machine while fast fading events can be additionally modelled as superimposed random variations that follow a given PDF for each state. Considering an arbitrary time instant $t$ and assuming that the transmitted signal has unitary amplitude ${ }^{1}$, the overall PDF describing the received signal amplitude, called below $R(t)$, can be written as:

$$
p_{R}(r)=\sum_{k=1}^{N} \Pi_{k} \cdot p_{R, k}(r)
$$

being $N$ the number of states, $\Pi_{k}$ the absolute probability of being in the state $k$ (that can be easily obtained from the State Transition Matrix $S=\left[p_{i j}\right]$, containing in each element the probability of transition from the state $i$ to the state $j$ ) and $p_{R, k}(r)$ the PDF associated to the fast fading within state $k$.

The LOS state is characterised by a Rician PDF of the following form:

$$
p_{R}(r)=\frac{r}{\sigma^{2}} \cdot \exp \left(-\frac{r^{2}+z^{2}}{2 \sigma^{2}}\right) \cdot I_{0}\left(\frac{r \cdot z}{\sigma^{2}}\right), \quad r \geq 0
$$

being $I_{0}$ the zero-order modified Bessel function of the first kind, $z$ the amplitude of the line-of-sight component and $\sigma^{2}$ the power of the real part or the imaginary part of the scattered component.

The Shadowed state is characterised by a Suzuki PDF, [10]. The Suzuki process is a product process of a Rayleigh process and a Lognormal (LN) process, [11]-[12]. The slow signal

${ }^{1}$ Under this hypothesis, the received signal amplitude, $R(t)$, corresponds to the amplitude of the fading term, $|A(t)|$, i.e. $R(t)=|A(t)|$. 
fading is, in this case, modelled by the Lognormal process taking the slow time variation of the average local received power into account. The Rayleigh process models, instead, the fast fading. The Suzuki PDF can be expressed as follows, [13]:

$$
\begin{aligned}
p_{R}(r)= & \int_{0}^{+\infty}\left[\frac{r}{\sigma_{r a y}^{2} L^{2}} \cdot \exp \left(-\frac{r^{2}}{2 \sigma_{r a y}^{2} L^{2}}\right)\right] \\
& {\left[\frac{1}{\sqrt{2 \pi} \phi \sigma_{l n} L} \cdot \exp \left\{-\frac{1}{2}\left(\frac{\ln (L)-\phi \mu_{l n}}{\phi \sigma_{l n}}\right)^{2}\right\}\right] d L }
\end{aligned}
$$

wherein the first term represents the conditional joint Lognormal and Rayleigh PDF while the second term is the Lognormal PDF which characterises the random variable $L$. Moreover, $\phi=\ln 10 / 20$, and $\mu_{l n}$ and $\sigma_{l n}$ are the mean and standard deviation, respectively, of the associated Gaussian distribution in $\mathrm{dB}$ unit.

Finally, the Blocked state is characterised by no signal availability. A different set of channel parameters can be derived for each of the two environments considered next, namely highway and suburban.

How the synchronization among users is managed in such a context, it is not in the scope of this investigation.

\section{Resource Allocation Mechanism}

In the proposed system, the resource allocation is done frame by frame; this means that the duration of a superframe is supposed to be equal to that of a frame. Each frame is divided in "logical" timeslots which correspond to a pair of frequency and time values. The bandwidth and duration of all timeslots are assumed equal. The first logical half of each frame is dedicated to the transmission while the second one to the cooperation. However, transmission slots come before cooperation ones in time because cooperators have to receive informative packets by active users in order to be able to cooperate.

The adopted resource allocation mechanism is a fair procedure, a "round robin" one, in which the same number of timeslots is assigned to users within the group of active users and within the group of cooperators. It is worth noting that:

- the number of timeslot assigned to the two groups can be different, depending on the number of users which demand to transmit in that particular frame;

- the number of users per group can change frame by frame;

- the specific users which belong to two groups can change frame by frame.

Moreover, the association between cooperators and active users is made analysing the distance matrix, $D$, whose dimensions are $N_{u} \times N_{u}$ with $N_{u}$ equal to the total number of sources considered:

$$
D=\left[\begin{array}{cccc}
d_{11} & d_{12} & \ldots & d_{1 N_{u}} \\
d_{21} & d_{22} & \ldots & d_{2 N_{u}} \\
\vdots & \vdots & \ddots & \\
d_{N_{u} 1} & d_{N_{u} 2} & \ldots & d_{N_{u} N_{u}}
\end{array}\right]
$$

where the elements $d_{i j}$ (with $i, j=1 \ldots N_{u}$ ) correspond to the distance values between each pair of users belonging to the overall group of considered sources. The choice is performed every frame, by selecting the closest one or two cooperators to each active user, which do not go beyond a predetermined cooperation threshold within the considered coverage area. The cooperation threshold is, therefore, the threshold beyond which two users cannot cooperate each other. In the simulation sessions, this has been fixed equal to $2 \mathrm{Km}$ considering a $5 \mathrm{Km}$ square coverage area. The selection of one or two cooperators, instead, depends on the assumed cooperation level which is the maximum number of cooperators fixed a priori in the system.

\section{System Performance}

In order to show the effectiveness of the proposed model and the benefits of the cooperation, several computer simulations have been performed. The considered source model parameters are those which have been defined in Table I while the other simulation parameters are defined in the following Table II. System performance has been calculated in terms of aggregated average throughput for different source loads, as shown in the following graphs.

The average throughput for the $k$-th active user, $\bar{R}_{k}$, (with $k=1 \ldots N_{u}$ ) is given by the following expression:

$$
\bar{R}_{k}=\frac{R_{k}}{N_{\text {rxframe }}}
$$

where $N_{\text {rxframe }}$ is the overall number of received frames in a simulation session while $R_{k}$ is the overall achieved throughput for the $k$-th active user which can be written as:

$$
R_{k}=\sum_{j=1}^{N} P_{c_{\text {tslot }} j} \cdot \frac{N_{\text {bit } / \text { tslot }}}{t_{\text {frame }}}
$$

being $P_{c_{\text {tslot }} j}$ the probability of correct reception of the $j$-th timeslot, with $j$ from 1 to $N$ which represents the number of timeslots in which the $k$-th active user has transmitted frame by frame, $N_{\text {bit/sslot }}$ the number of bits in a timeslot ${ }^{2}$ and $t_{\text {frame }}$ the duration of a frame.

The probability of correct reception of a timeslot can be derived from the bit error probability, $P_{e b}$, calculated at Physical layer, as follows:

$$
P_{c_{\text {tslot }}}=\left(1-P_{e b}\right)^{N_{b i t / t s l o t}}
$$

The value of $P_{e b}$ depends on the adopted code rate, the $E_{b} / N_{0}$ value, the considered environment (highway and suburban), the conditions of the LMS channel and the number of cooperators involved in the cooperation. The higher the cooperation level, the lower is the value of $P_{e b}$ for a determined environment.

Finally, the aggregate average throughput, $\bar{R}_{\text {aggr }}$, is defined as the sum of average throughput achieved by all users which have transmitted informative packets in a simulation session:

$$
\bar{R}_{\text {aggr }}=\sum_{k=1}^{N_{u}} \bar{R}_{k}
$$

\footnotetext{
${ }^{2}$ This value represents the ATM traffic burst size with $N_{\text {atm }}=1$, i.e. 53 Bytes which are equivalent to 424 bits.
} 


\begin{tabular}{|c|c|}
\hline$N_{u}$ & 20 \\
\hline$N_{\text {bit } / \text { tslot }}$ & 424 \\
\hline$t_{\text {frame }}$ & $0.0265 \mathrm{~s}$ \\
\hline$N_{\text {timetslot } / \text { frame }}$ & 24 \\
\hline$N_{\text {carrier } / \text { frame }}$ & 8 \\
\hline$N_{\text {tslot } / \text { frame }}$ & 192 \\
\hline$E_{b} / N_{0}$ & $2 \mathrm{~dB}$ \\
\hline
\end{tabular}

TABLE II

SIMULATION PARAMETERS

The set of parameters used in simulations is reported in Table II.

The number of timeslots "in time" per frame, $N_{\text {timetslot/frame, }}$ i.e. the number of time portions available for each carrier per frame, is obtained through the following calculation, considering that each source emits packets at the peak rate $\rho:$

$$
N_{\text {timetslot } / \text { frame }}=\rho \cdot \frac{t_{\text {frame }}}{N_{\text {bit } / \text { slot }}}
$$

The total number of logical timeslot per frame is, therefore, given by:

$$
N_{\text {tslot } / \text { frame }}=N_{\text {timetslot } / \text { frame }} \cdot N_{\text {carrier } / \text { frame }}
$$

\section{A. No cooperation case}

This section presents some results concerning the no cooperation case. With the term "no cooperation", it is meant the case where sources use the whole frame for transmitting their informative packets and do not require the adoption of a cooperation scheme.

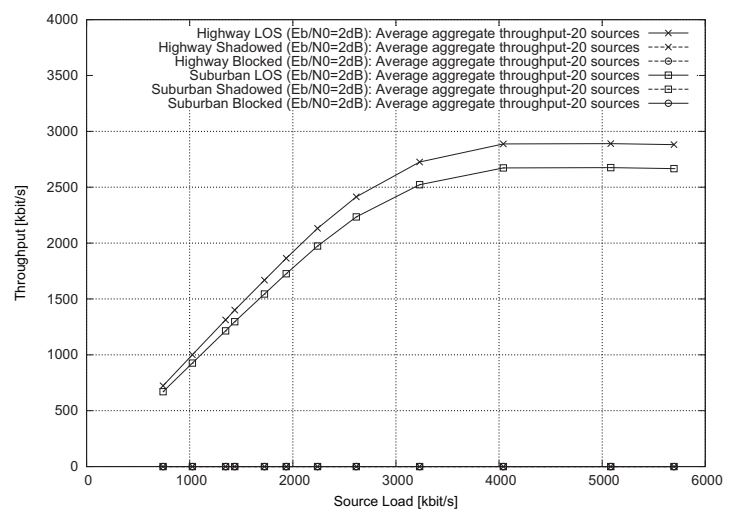

Fig. 3. Aggregate average throughput - No cooperation case - HIGHWAY and SUBURBAN environments - LOS, Shadowed and Blocked states - 20 sources $-E_{b} / N_{0}=2 d B$

In Fig. 3, the aggregate average throughput curves for two considered environments, highway and suburban, and for three different LMS channel states are reported. The graph shows as without the adoption of cooperation only if the channel is in LOS conditions, the system achieves high values of throughput which approach the system capacity given, in this case, by:

$$
C_{\text {nocoop }}=\rho \cdot N_{\text {carrier } / \text { frame }}=3072 \mathrm{kbit} / \mathrm{s}
$$

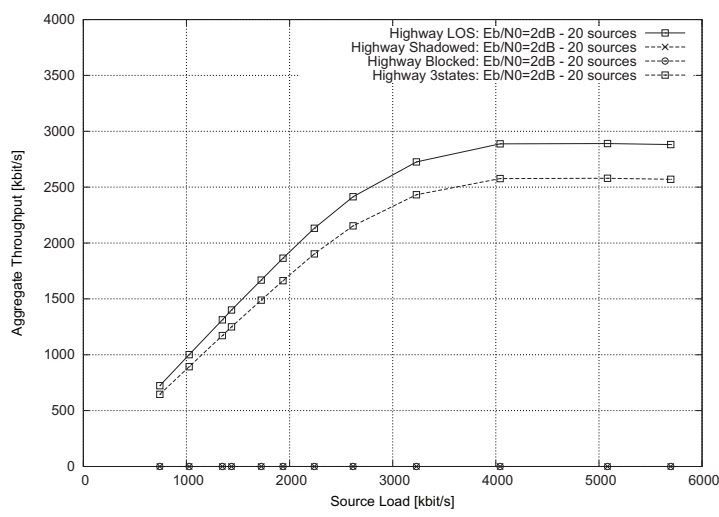

Fig. 4. Aggregate average throughput - No cooperation case - HIGHWAY environment - LOS, Shadowed, Blocked and 3 states -20 sources $-E_{b} / N_{0}=$ $2 \mathrm{~dB}$

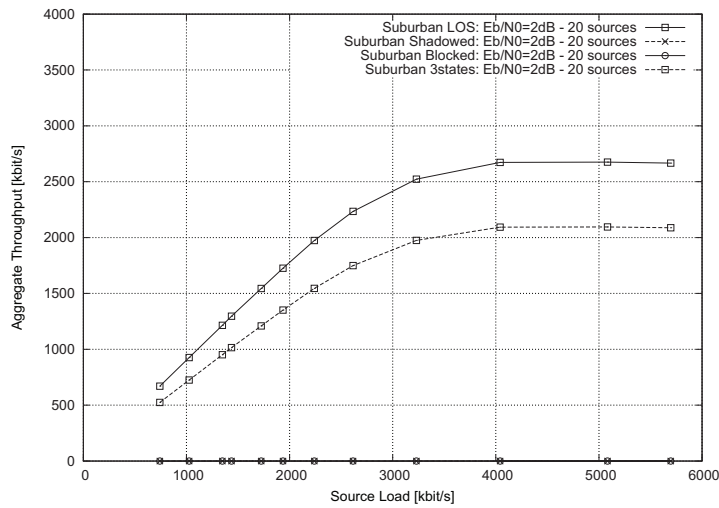

Fig. 5. Aggregate average throughput - No cooperation case - SUBURBAN environment - LOS, Shadowed, Blocked and 3 states - 20 sources $-E_{b} / N_{0}=$ $2 \mathrm{~dB}$

whereas, if the channel is in Shadowed or in Blocked state, the system capacity of transmitting packets is close to zero. Fig. 4 and Fig. 5 show the throughput performance for the two environments separately, introducing in each graph also the average curve, obtained computing the aggregated throughput averaged over sessions where the channel state was only LOS, Shadowed and Blocked. The average curve is close to the LOS one because the LOS state is the state with the highest absolute probability $(89.22 \%$ in the highway environment and $78.31 \%$ in the suburban environment).

\section{B. Cooperation case}

In the following, the results achieved in the cooperation case are reported and analysed. The cooperation case encompasses three different cooperative cases:

- no cooperators (cooperation level $=0$ )

- 1 cooperator (cooperation level $=1$ )

- 2 cooperators (cooperation level=2)

The 1 cooperator and 2 cooperators cases envisage the possibility for each active user to have up to one or two cooperators, depending on the assumed cooperation level value. Also the "no cooperators" is considered as cooperation case because, although there are no active cooperators, only half frame is 


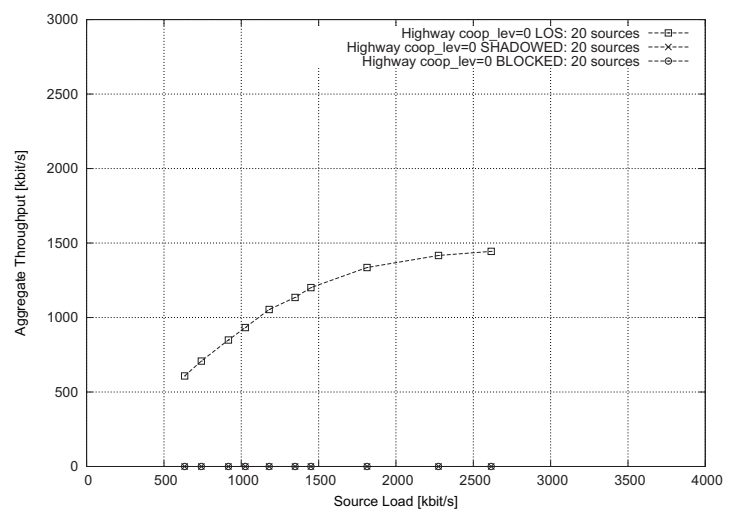

Fig. 6. Aggregate average throughput - Cooperation case, no cooperators HIGHWAY environment - LOS, Shadowed and Blocked states - 20 sources $E_{b} / N_{0}=2 \mathrm{~dB}$

used for transmitting informative packets.

Fig. 6 shows the aggregate average throughput curves in the no cooperators case, considering the highway environment and each of the three channel states. As already seen above for the no cooperation case, only if the channel is in LOS conditions, the system achieves high values of throughput which approach the cooperative system capacity given here by:

$$
C_{\text {coop }}=\frac{\rho}{2} \cdot N_{\text {carrier } / \text { frame }}=1536 \mathrm{kbit} / \mathrm{s}
$$

because only half of every frame is used for the transmission of active users' packets. Also in this case, if the channel is in Shadowed or in Blocked state, the cooperative system capacity is close to zero.

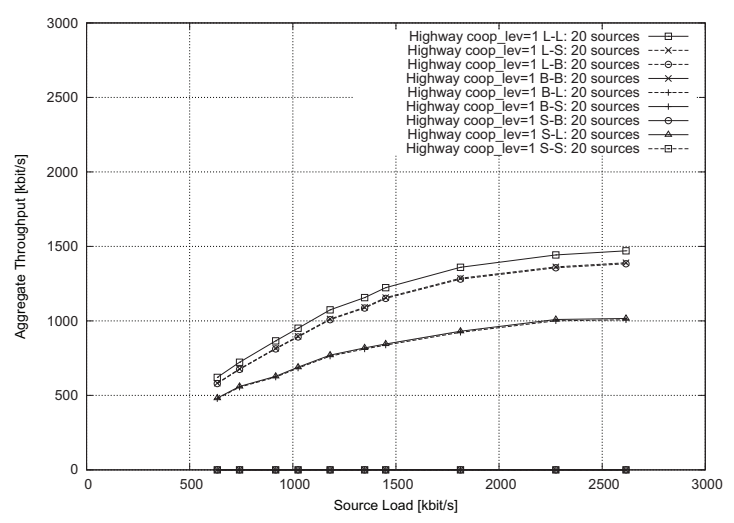

Fig. 7. Aggregate average throughput - Cooperation case, 1 cooperator HIGHWAY environment - LOS, Shadowed and Blocked states - 20 sources $E_{b} / N_{0}=2 \mathrm{~dB}$

Fig. 7, instead, considers the 1 cooperator case and shows the throughput performance considering all possible combinations of the satellite channel conditions faced by active user and cooperator, in the highway environment. In the graph, the legend reports for each curve the expression " $X-Y$ " where $X$ and $Y$ are the channel state encountered by the active user and the cooperator ${ }^{3}$, respectively. In those cases in which at

\footnotetext{
3"L" stands for LOS, "S" for Shadowed and "B" for Blocked.
}

least the active user or the cooperator see the channel in LOS conditions, the system is able to transmit, achieving the best values of throughput when both active user and cooperator face the channel in LOS state. Hence, the number of scenarios wherein the system can transmit data increases when one cooperator is present.

Similar results can be assessed analysing the 2 cooperators case. Fig. 8, Fig. 9 and Fig. 10 report the aggregate average throughput curves achieved in the highway environment when each active user sees the channel in LOS state, Shadowed state and Blocked state, respectively. All possible combinations of the channel conditions faced by the active user and by the 2 cooperators are considered. Also in this case, in the graphs, the legend reports for each curve the expression " $X-Y-Z$ " where $X, Y$ and $Z$ are the channel state encountered by the active user, the first cooperator and the second cooperator ${ }^{3}$, respectively. In all cases in which at least either the active user or one of cooperators sees the channel in LOS conditions, the system is able to transmit, achieving the best values of throughput when the active user and at least one of cooperators are in LOS state. Hence, if 2 cooperators are involved in the cooperation, the number of scenarios wherein the system can transmit packets increases considerably compared to the no cooperators case.

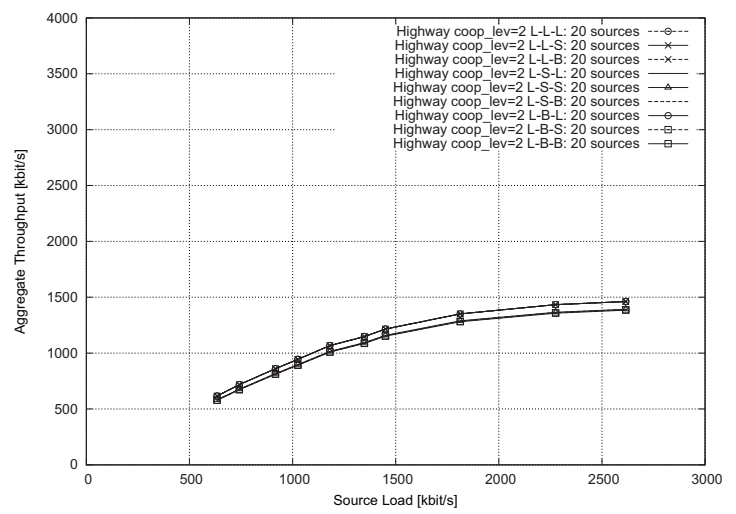

Fig. 8. Aggregate average throughput - Cooperation case, 2 cooperators HIGHWAY environment - LOS state -20 sources $-E_{b} / N_{0}=2 \mathrm{~dB}$

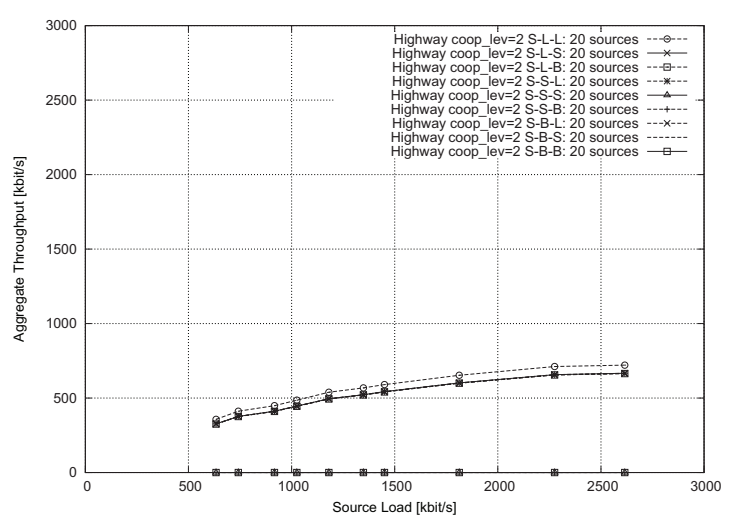

Fig. 9. Aggregate average throughput - Cooperation case, 2 cooperators HIGHWAY environment - Shadowed state - 20 sources $-E_{b} / N_{0}=2 \mathrm{~dB}$ 


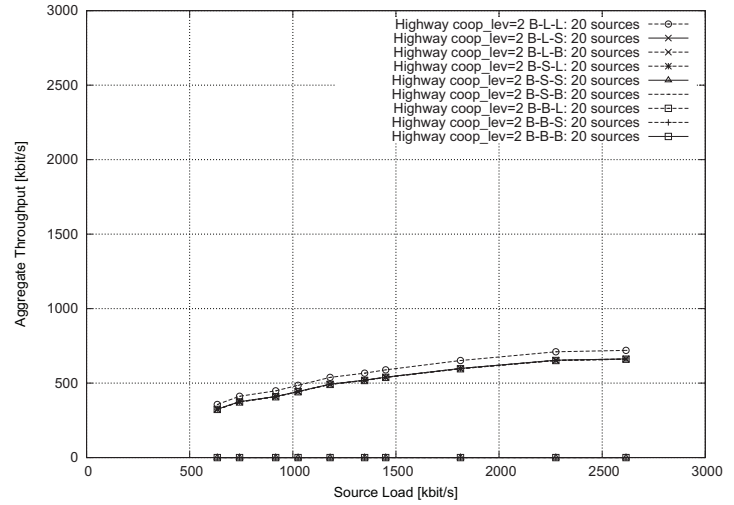

Fig. 10. Aggregate average throughput - Cooperation case, 2 cooperators HIGHWAY environment - Blocked state - 20 sources $-E_{b} / N_{0}=2 \mathrm{~dB}$

Fig. 11, Fig. 12 and Fig.13 show the comparison among the analysed cooperation cases (no cooperators, 1 cooperator and 2 cooperators), and report the aggregate average throughput achieved in the highway environment when the satellite channel seen by sources is in LOS state, in Shadowed state and in Blocked state, respectively. All possible combinations of channel conditions seen by cooperators are taken into account both in the case of 1 cooperator and in that of 2 cooperators.

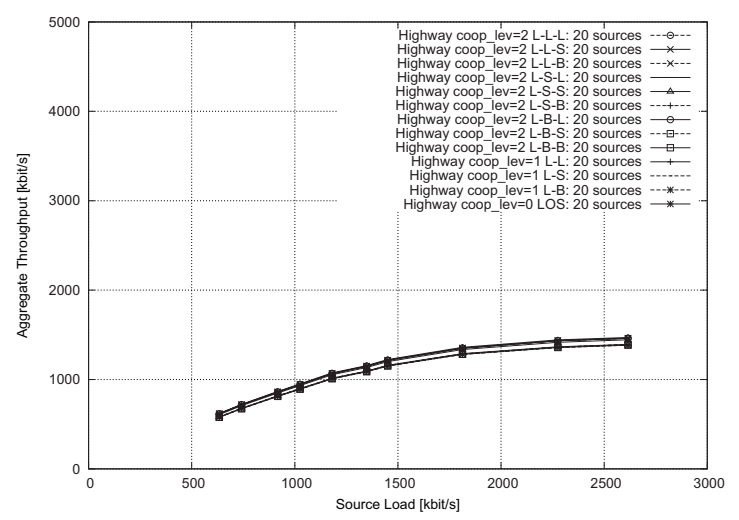

Fig. 11. Aggregate average throughput comparison among the cooperation cases (no cooperators, 1 cooperator, 2 cooperators) - HIGHWAY environment, LOS state - 20 sources - $E_{b} / N_{0}=2 \mathrm{~dB}$

It is interesting to note that in Fig. 11 (sources seeing the channel in LOS state) the curves are very close, regardless of the number of involved cooperators. This happens because active users encounter good channel conditions and their performances are less affected by cooperation. The improvements of performance are, instead, remarkable in the other two cases shown in Fig. 12 and Fig. 13 (sources facing the channel in Shadowed and in Blocked state). In fact, in these cases, the retransmission operated by cooperators becomes fundamental, almost essential, in order to not to lose some pieces of information belonging to the active users.

The same considerations can be done observing Fig. 14,

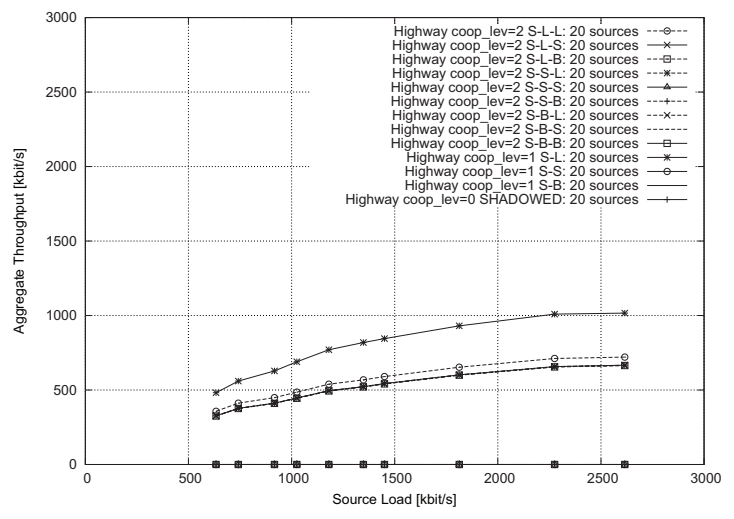

Fig. 12. Aggregate average throughput comparison among the cooperation cases (no cooperators, 1 cooperator, 2 cooperators) - HIGHWAY environment, Shadowed state -20 sources $-E_{b} / N_{0}=2 \mathrm{~dB}$

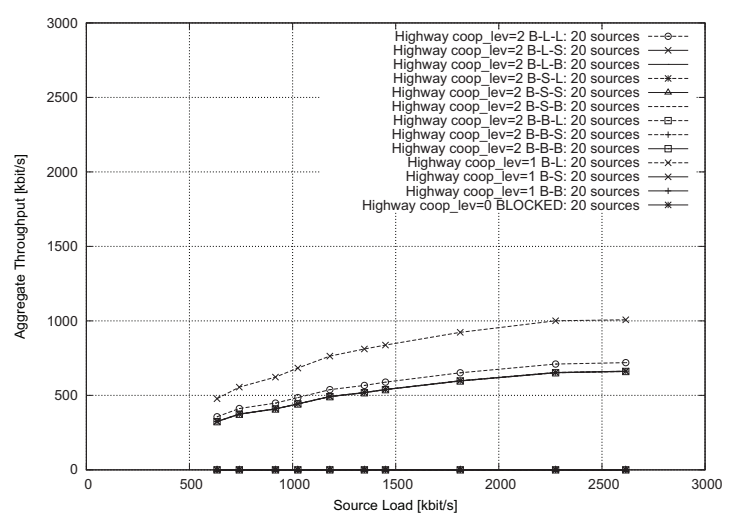

Fig. 13. Aggregate average throughput comparison among the cooperation cases (no cooperators, 1 cooperator, 2 cooperators) - HIGHWAY environment, Blocked state - 20 sources $-E_{b} / N_{0}=2 \mathrm{~dB}$

Fig. 15 and Fig.16 which show the aggregate average throughput achieved in the suburban environment when the satellite channel seen by sources is in LOS state, in Shadowed state and in Blocked state, respectively, for each of the cooperation cases (no cooperators, 1 cooperator and 2 cooperators) analysed. Finally, the last results are reported in Fig. 17 and in Fig. 18, where the throughput curves have been obtained computing the aggregated throughput averaged over sessions where the channel state was only LOS, Shadowed and Blocked, in the highway and suburban environments, respectively. Through these graphs, the advantage of using the cooperation in such a context can be again emphasized.

\section{CONCLUSION}

This paper has presented an assessment of cooperation effects at MAC layer in DVB-RCS systems, through the implementation of a modified resource allocation mechanism for satellite users working in a land-vehicular scenario. The achieved results in terms of aggregated average throughput, calculated for different source loads, have shown promising outcomes. In fact, the use of cooperation can allow improving system performance depending on the number of cooperators 


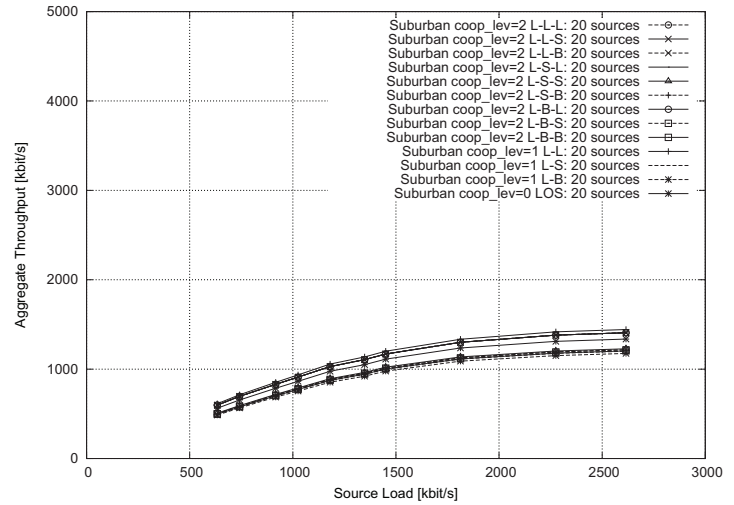

Fig. 14. Aggregate average throughput comparison among the cooperation cases (no cooperators, 1 cooperator, 2 cooperators) - SUBURBAN environment, LOS state - 20 sources - $E_{b} / N_{0}=2 \mathrm{~dB}$

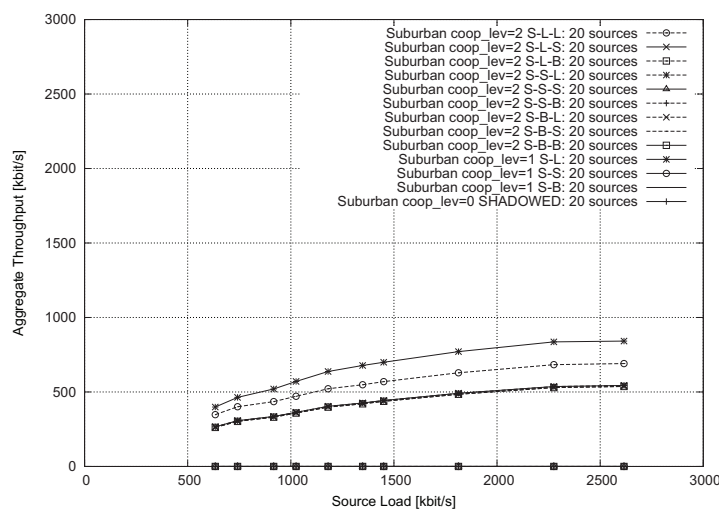

Fig. 15. Aggregate average throughput comparison among the cooperation cases (no cooperators, 1 cooperator, 2 cooperators) - SUBURBAN environment, Shadowed state -20 sources $-E_{b} / N_{0}=2 \mathrm{~dB}$

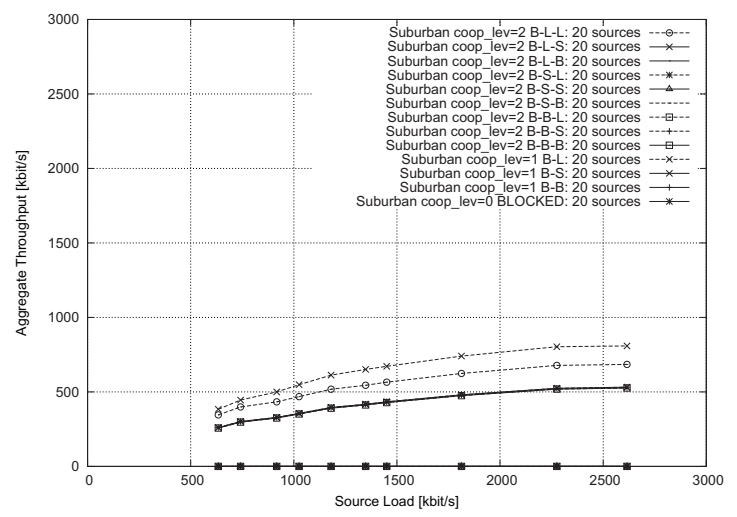

Fig. 16. Aggregate average throughput comparison among the cooperation cases (no cooperators, 1 cooperator, 2 cooperators) - SUBURBAN environment, Blocked state -20 sources $-E_{b} / N_{0}=2 \mathrm{~dB}$

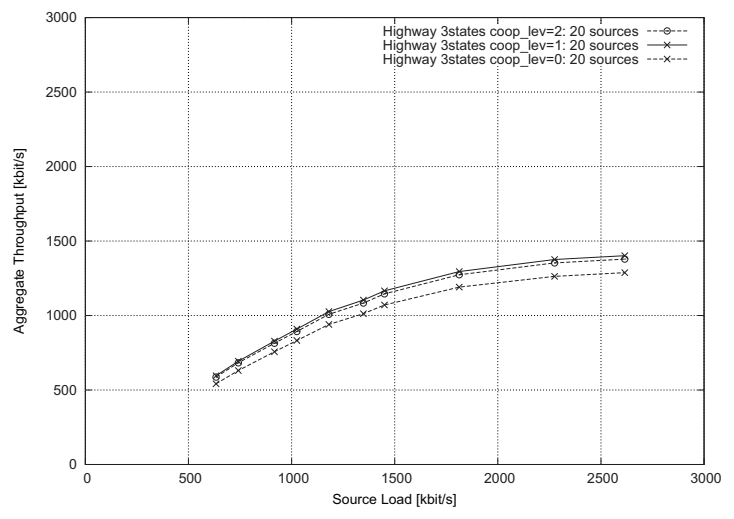

Fig. 17. Aggregate average throughput comparison among the cooperation cases (no cooperators, 1 cooperator, 2 cooperators) - HIGHWAY environment, 3 states - 20 sources $-E_{b} / N_{0}=2 \mathrm{~dB}$

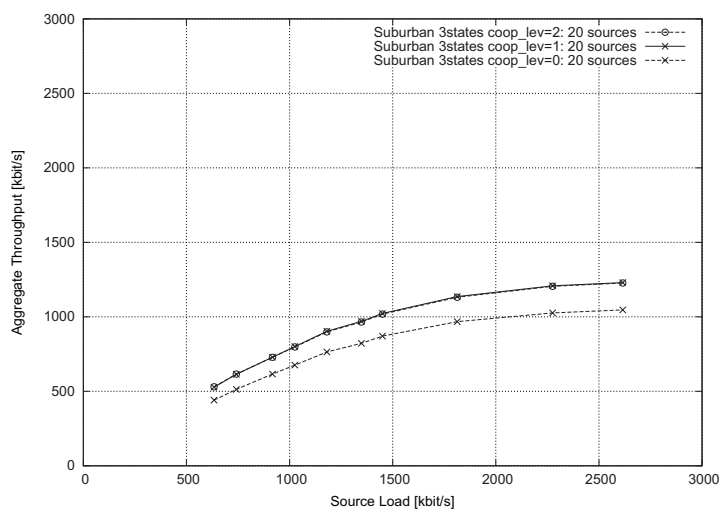

Fig. 18. Aggregate average throughput comparison among the cooperation cases (no cooperators, 1 cooperator, 2 cooperators) - SUBURBAN environment, 3 states - 20 sources - $E_{b} / N_{0}=2 \mathrm{~dB}$

considered and the different channel conditions which they are subject to. In particular, it can allow increasing the number of scenarios wherein the system can transmit data packets compared to the absence of cooperation. For these reasons, it is worth investigating further on the adoption of the cooperation in such critical satellite environments in order to deepen some related aspects not considered in this work.

\section{REFERENCES}

[1] ETSI EN 301790 v 1.4.1, Digital Video Broadcasting (DVB): Interaction channel for satellite distribution systems, 2005-09.

[2] A. Ribeiro, G.B. Giannakis, Fixed and Random Access Cooperative Networks, EURASIP Newsletter, pp. 3-24, March 2006.

[3] A. Nosratinia, T.E. Hunter, A. Hedayat, Cooperative Communication in Wireless Networks, IEEE Communications Magazine, Vol. 42, No. 10, pp. 74-80, October 2004.

[4] T.B. Fowler, A Short Tutorial on Fractals and Internet Traffic, The Telecommunications Review, Vol. 10, pp. 1-15, 1999.

[5] M.J. Fisher, D. Gross, D. M. Bevilacqua Masi, J.F. Shortle, Analyzing the Waiting Time Process in Internet Queueing Systems With the Transform Approximation Method, The Telecommunications Review, Vol. 12, pp. 21-32, 2001.

[6] T. Bohnert, E. Monteiro, A Comment on Simulating LRD Traffic with Pareto ON/OFF Sources, CoNEXT'05, October 2005, Toulouse, France.

[7] ETSI TR 101790 v 1.3.1, Digital Video Broadcasting (DVB); Interaction channel for Satellite Distribution Systems; Guidelines for the use of EN 301 790, 2006-09. 
[8] Final Draft ETSI EN 301790 v 1.5.1, Digital Video Broadcasting (DVB): Interaction channel for satellite distribution systems, 2009-01.

[9] S. Scalise, H. Ernst, G. Harles, Measurement and Modelling of the Land Mobile Satellite Channel at Ku-Band, IEEE Transactions on Vehicular Technology, Vol. 57, No. 2, pp. 693-703, March 2008.

[10] H. Suzuki, A Statistical Model for Urban Radio Propagation, IEEE Transactions on Communications, Vol. 25, No. 7, pp. 673-680, July 1977.

[11] H. Flemming, M.I. Finn, Mobile Fading-Rayleigh and Lognormal Superimposed, IEEE Transactions on Vehicular Technology, Vol. 26, No. 4, pp. 332-335, November 1977.

[12] M. Pätzold, Mobile Fading Channels, Wiley, January 2002.

[13] H.P. Lin, M.C. Tseng, D.B. Lin, Performance analysis of M-ary PSK adaptive modulation system over Rayleigh-lognormal fading channel, IEEE Vehicular Technology Conference Spring (VTC2005Spring), May 2005, Stockholm, Sweden.

[14] X.Pan, L.Wei, Resource allocation in variable bandwidth MF-TDMA satellite communications, IEEE International Conference on Signal Processing ICSP2006, November 2006, Guilin, China. 